\title{
Percutaneous posterior tibial nerve stimulation for the treatment of the overactive bladder in children: Is it effective?
}

\author{
Hisham Ibrahim, Ahmed Mahmoud Shouman, Waseem Ela, Waleed Ghoneima, Ahmed Ismail Shoukry, \\ Mohammed ElSheemy, Hani Morsi, Hesham Badawy, Mohamed Eissa and Sameh Kotb* (0)
}

\begin{abstract}
Background: To assess the efficacy and durability of percutaneous posterior tibial nerve stimulation for the treatment of the overactive bladder in children.

Results: Twenty patients with a mean age of $7 \pm 3.3$ years were included in the study. Subjective success was found in 12 patients (60\%) who considered their situation to be better and requested to continue therapy to maintain the response which was considered as a therapeutic success, while eight patients (40\%) patients chose not to continue therapy because they did not have symptomatic improvement.

Conclusion: Posterior tibial nerve stimulation is an effective, minimally invasive method for treatment and is easily applied to induce improvement in overactive bladder symptoms and objective urodynamic changes with negligible side effects.
\end{abstract}

Keywords: Posterior tibial nerve, Electric stimulation, Urodyanmic, Irritative symptoms, Overactive bladder

\section{Background}

Overactive bladder (OAB) symptoms (urgency, frequency, nocturia, and urge incontinence) are frequent complaints in urology clinics. The cause of detrusor overactivity (DO) in most cases is idiopathic. Sleep disturbance and psychological distress also affect those patients [1].

There are a variety of treatment options available for the treatment of OAB. The mainstay of treatment currently is the use of anticholinergic drugs. Many patients find these dugs effective; however, they suffer from its side effects [2]. Second-line treatment options include the use of intravesical botulinium toxin, sacral nerve stimulation, and percutaneous posterior tibial nerve stimulation [3].

Percutaneous posterior tibial nerve stimulation (PTNS) is a technique that was developed a long time ago, but

*Correspondence: dr_samehkotb@kasralainy.edu.eg

Urology Department, Aboul-Riche Children's Hospital, Kasr Al-Ainy Hospitals, Cairo University, Cairo, Egypt it does not appear to have become widely adopted into clinical practice. It is currently gaining much interest with improved equipment and marketing. Urgent $\mathrm{PC}^{\circledR}$ represents an improved neuromodulation technique that is minimally invasive and easy to perform. Stimulation is carried out through a percutaneously placed needle in 12 weekly sessions of $30 \mathrm{~min}$ each [4].

It seems reasonable to consider PTNS when conservative and non-surgical treatment options are being encouraged for the management. Knowledge of how PTNS work came from studying sacral nerve stimulation. However, the exact mechanism of action is still not understood. There may be different modes of action in different clinical conditions. Some may involve the gate theory, by restoring control at the spinal segment gate as well as some supraspinal sites. Others may involve restoration both centrally and peripherally of the balance between inhibitory and excitatory control systems [5].

Limited side effects of PTNS are present related to discomfort at the insertion site of the needle. The need for maintenance treatment after the initial 12 weeks course 
of therapy may be limited if the patient did not improve because he will not continue therapy if there is no initial improvement [6].

We aim in this study to assess the efficacy and durability of PTNS for the treatment of the overactive bladder in children.

\section{Methods}

This prospective non-randomized case series was done between January 2016 and January 2018. Twenty patients with frequency, urgency, nocturia, and urge incontinence were included in this study. Patients with urinary tract infection (UTI), upper tract affection, infravesical obstruction (posterior urethral valve), or neurological diseases were excluded from this study.

All patients were subjected to full history taking including urological symptoms (frequency, urgency, nocturia, and urge incontinence), medical history including drugs as anticholinergic, and previous pelvic or urological procedure. The bladder diary was recorded including frequency, nocturia, number of leakage episodes (urge incontinence), and nocturnal enuresis. Complete physical examination including neurological assessment of perianal sensation and patient gait was performed. Laboratory investigations in the form of renal function tests (urea and creatinine), urine analysis, and urine culture and sensitivity are carried out if needed.

Radiological investigations were done in the form of abdomino-pelvic ultrasound: ascending and micturating cystourethrogram. Urodynamic evaluation reporting bladder capacity, compliance, sensations, presence of detrusor overactivity, leakage, urine flow, and residual urine was done.

Patients were subjected to PTNS. Patients should experience a tolerable but not painful sensation particularly in the region of the large toe. If the large toe does not curl or there is pain at the insertion site, the stimulation device should be switched off and the needle should be repositioned.

All patients were asked to evacuate their bladder before starting the treatment session to ensure that they are relaxed and comfortable during session. After hand washing, sterile disposable gloves are worn before starting the session. The area surrounding the medial malleolus is cleaned by using sterilizing solution.

The patient sits comfortably with a needle electrode inserted into the lower inner aspect of the leg slightly cephalic to the medial malleolus after leg elevation.

A surface electrode (grounding pad) is placed over the medial aspect of the calcaneus on the same leg. An external pulse generator which delivers an adjustable electrical pulse that travels to the sacral plexus via the tibial nerve is then connected to the needle electrode. Regulation of the bladder and pelvic floor function are among other functions of the sacral nerve plexus. There is often an involuntary toe flex or an extension of the entire foot with correct placement of the needle electrode. However, for some patients, the correct placement and stimulation may only result in a mild sensation in the ankle area or across the sole of the foot. The treatment protocol requires 12 sessions for 12 weeks, 30 min per each.

Patients with symptom improvements after initial 12 treatments may choose to continue treatment at individually based greater intervals for sustained relief $O A B$ symptoms and fear of relapse. Follow-up is done after 3 months by urodynamic test and evaluation of symptomatic improvement.

Subjective success is defined as the patients' positive response resulting in the request for continuing treatment for sustained relief OAB symptoms. Objective response was based on bladder diary and urodynamic parameters. Objective success was defined as $50 \%$ reduction in symptoms, statistically significant improvement in urodynamic parameters.

Numerical data were statistically described in terms of mean \pm standard deviation $( \pm S D)$ and range, while categorical data were described using frequencies (number of cases) and percentages. All statistical calculations were done using computer program SPSS (Statistical Package for the Social Science; SPSS Inc., Chicago, IL, USA) release 15 for Microsoft Windows.

\section{Results}

Twenty patients were complaining of $\mathrm{OAB}$ symptoms (urgency, frequency, nocturia, nocturnal enuresis, and urge incontinence). Patient demographics are listed in Table 1.

Subjective success was found in twelve patients $(60 \%)$ considered their situation better and requested to continue therapy to maintain the response which was considered as a therapeutic success, while eight patients (40\%) did not choose to continue therapy because they did not have symptomatic improvement.

As regards the objective success, 12 patients out of 20 (60\%) showed improvement in their daytime frequency. Eleven patients out of $20(55.0 \%)$ showed improvement in their urgency. Ten out of 16 patients (63.0\%) had

\section{Table 1 Patients demographics}

\begin{tabular}{ll}
\hline Patient number & $\mathbf{2 0}$ \\
\hline Patient sex & \\
Male & $8(40 \%)$ \\
Female & $12(60 \%)$ \\
Mean $\pm S D$ age in years & $7 \pm 3.3$ years \\
\hline
\end{tabular}


Table 2 Symptomatic evaluation

\begin{tabular}{lll}
\hline Symptom & Improved (\%) & $\begin{array}{l}\text { Not } \\
\text { improved } \\
\text { (\%) }\end{array}$ \\
\hline Urgency & 55 & 45 \\
Daytime frequency & 60 & 40 \\
Urge incontinence & 53 & 47 \\
Nocturnal enuresis & 63 & 37 \\
Overall symptomatic improve- & 60 & 40 \\
ment & & \\
\hline
\end{tabular}

Table 3 Overall improvement in bladder over activity

\begin{tabular}{lcc}
\hline & No. & Improved \\
\hline High amplitude & 7 & 3 \\
Low amplitude & 13 & 11 \\
Total & 20 & 14 \\
\hline
\end{tabular}

nocturnal enuresis showed improvement in their nocturnal enuresis. Eight out of 15 patients (53.0\%) had urge incontinence showed improvement in their incontinence. Average incontinence episodes varied from 5 to 10 times. Symptomatic evaluation is listed in Table 2.

Evaluation of urodynamic parameters before and after PTNS includes detrusor overactivities, bladder capacity, and bladder compliance. All the patients were suffering from involuntary detrusor contractions. Seven of them had high-amplitude detrusor contractions $\left(>40 \mathrm{~cm} \mathrm{H}_{2} \mathrm{O}\right)$, while 13 had low amplitude detrusor contractions. Three out of seven patients (42.8\%) with high amplitude had their involuntary contractions disappear, two patients had low amplitude, and two still have high-amplitude detrusor contractions. Eleven out of 13 (84.6\%) patients with low amplitude had their contractions disappear.

Overall improvement, 14 out of $20(70 \%)$ had their contractions disappeared while six (30\%) still have involuntary detrusor contractions. Overall improvement in bladder over activity is listed in Table 3.

There was a highly significant difference between bladder capacities before and after taking the sessions $(p=0.001)$. The bladder capacities were $184.5 \pm 59.14$ versus $259.5 \pm 77.22$ for the before and after sessions, respectively.

Eighteen patients had hypocompliant bladder, seven patients mild hypocompliant bladder, five patients moderate hypocompliant bladder, and six patients severe hypocompliant bladder. Four out of seven patients (57.1\%) with mild hypocompliance showed improvement in their compliance. One out of five (20\%) patients with moderate hypocompliance showed improvement.
However, patient with severe hypocompliance did not improve. No adverse side effects were noted during PTNS except transient pain at the site of needle insertion.

\section{Discussion}

Overactive bladder is a common condition that has an impact on physical, psychological, and social well-being of the patients [7]. The cause of their symptoms is related to DO which may be idiopathic with no obvious underlying neurological abnormality in most cases [8].

The first line of treatment for overactive bladder is anticholinergic therapy but it is limited by side effects. Another modality of treatment is neuromodulation which is an effective treatment [9].

Posterior tibial nerve is a mixed nerve containing sensory and motor nerve fibers [10]. PTNS is one of the least invasive forms of neurostimulation, since posterior tibial nerve shares the sacral roots with bladder afferent [11].

In our study, patients were chosen to have symptoms of overactive bladder (frequency, urgency, nocturnal enuresis, and urge incontinence) with no underlying neurological deficit, no urinary tract infection or upper tract affection.

We used weekly PTNS for 12 weeks that lasts for $30 \mathrm{~min}$ in each session. The protocol we used was different from the study done by Van der Pal [6] in which participants received PTNS three times a week for 4 weeks. It was also different from Van Balken [12] in which there is maintenance of therapy, and the necessity of maintenance therapy was evaluated by means of a 6 -week pause of therapy in successfully treated patients, leading to over $50 \%$ worsening of main symptoms.

Restarting PTNS afterward improved complaints to the level present before stopping. Capitanucci et al. [13] reported that repeating PTNS cycles and eventually using chronic monthly stimulation are necessary to maintain results.

Remarkable clinical results were obtained in $60 \%$ of our patients who reported a significant subjective success which was comparable to Vandoninck et al. [10] who reported that the subjective response was 64\% (58/90). In another study by Van Balken et al. [12], the subjective response was $55 \%$ (defined as a patient request for continuous chronic treatment to maintain the response).

Hoebeke et al. [4] reported improvement in daytime frequency in 16 out of 19 (84\%), urgency in 17 out of 28 (61\%), and daytime incontinence in 16 out of 23 (70\%). In the De Gennaro et al. [14] study, the improvement in nocturnal enuresis in five out of eight patients, daytime frequency urgency in five out of 10 patients, and incontinence in five out of eight patients was comparable to our results. 
Objective success in our study was defined as 50\% reduction in symptoms, statistically significant improvement in urodynamic parameters as in Van Balken et al. [12], while Vandoninck et al. [10] reported that objective response was defined as reduction in number of urinary leakage episodes of $50 \%$ or more per $24 \mathrm{~h}$.

In comparison with De Gennaro et al. [14], urodynamic showed normalization of cystometric bladder capacity in $62.5 \%$ with no more unstable contractions in those who became continent and urodynamics showed an improved detrusor pressure at maximum flow $(p=0.009)$. In another study done by Hoebeke et al. [4], mean bladder capacity increased from 185.16 to $279.19 \mathrm{ml}$, and there was statistically significant increase in bladder capacity $(p$ $0.001)$.

In our study, no serious side effects were observed except for transient pain at the site of needle insertion that was the same as Capitanucci et al. [13] study which reported that no significant side effect was observed, while in the study of Hoebeke et al. [4], it was reported that only one of 32 children discontinued treatment because of needle fear, and another study done by Van der Pal [6] reported some bleeding or pain at insertion site and numbness sole of foot.

Follow-up was done after 3 months in the form of symptomatic evaluation using bladder diary and urodynamic test that was different from Capitanucci et al. [13] study in which follow-up is extended up to 2 years in the form of bladder diary and urine analysis and also different from Congregrado Ruiz [15], in which followup is done after 21 months while Van der Pal [6] study in which follow-up is done after 1 month.

A weak point in our study is that we had relative a smaller number of patients compared to other studies as in a study by Hoebeke et al. [4], which include 32 patients (17 boys, 15 girls) and in Capitanucci et al. [13] which include 44 children ( 25 female, 19 male), but we were among few studies that treat pediatric age group as a study by Doyle et al. [16] which included only eight children which also showed that PTNS is safe and well tolerated to children that had failed previous standard treatments for overactive bladder with minimal side effects as difficulties in maintaining weekly treatment schedule and these results are in agreement with our study.

\section{Conclusion}

PTNS is an effective, minimally invasive method for treatment and is easily applied induce improvement in overactive bladder symptoms and objective urodynamic changes with negligible side effects.
Abbreviations

OAB: overactive bladder; DO: detrusor overactivity; PTNS: percutaneous posterior tibial nerve stimulation; UTI: urinary tract infection; $\mathrm{CBC}$ : complete blood count; SD: standard deviation.

\section{Acknowledgements}

Not applicable.

\section{Authors' contributions}

$\mathrm{HI}$ collected the patient data, analyzed them, and followed the patients postoperatively. WE, WG, AIS, MES, HM, and HB analyzed patient data and followed the patients postoperatively. AM and ME gave idea and contributed to study design. SK drafted the article revising it critically for important intellectual content and wrote the paper with revision. All authors read and approved the final manuscript.

\section{Funding}

This study received no funding from any resource.

\section{Availability of data and materials}

The datasets used and/or analyzed during the current study are available from the corresponding author on reasonable request.

\section{Ethics approval and consent to participate}

This study was approved by the Research Ethics Committee of the Faculty of Medicine, Cairo University in Egypt. Ethics committee reference numbers is not available. All patients included in this study gave written informed consent to participate in this research.

\section{Consent for publication}

All patients included in this research gave written informed consent to publish the data contained within this study.

\section{Competing interests}

The authors declare that they have no competing interests.

Received: 26 October 2019 Accepted: 14 November 2019

Published online: 27 November 2019

\section{References}

1. Govier FE, Litwiller S, Kreder K et al (2001) Percutaneous afferent neuromodulation for the refractory overactive bladder; results of multicenter study. Urology 165:1193-1198

2. Brazelli M, Murray A, Fraser C (2006) Efficacy and safety of sacral nerve stimulation for urinary urge incontinence: a systematic review. J Urol 175:835-841

3. Casanova N, McGuire E, Fenner DE (2006) Botulinum toxin: a potential alternative to current treatment of neurogenic and idiopathic urinary incontinence due to detrusor overactivity. Int J Gynaecol Obstet 95:305-311

4. Hoebeke P, Renson C, Petillon L et al (2002) Percutaneous electrical nerve stimulation in children with therapy resistant nonneuropathic bladder sphincter dysfunction: a pilot study. J Urol 168:2605-2607

5. Van Balken MR, Vergunst $H$, Bemelmans BL (2007) Prognostic factors for successful percutaneous tibial nerve stimulation. Eur Urol 49:360-365

6. Van der Pal F, Van Balken MR, Heesakkers JP et al (2006) Percutaneous tibial nerve stimulation in the treatment of refractory overactive bladder syndrome: is maintenance treatment necessary? BJU Int 97(3):547-550

7. Correia Sofia, Dinis Paulo, Lunet Nuno (2009) Urinary incontinence and overactive bladder: a review. ArquiMed 23(1):13-21

8. Reeves P, Irwin D, Kelleher C et al (2006) The current and future burden and cost of overactive bladder in five European countries. Eur Urol 50:1050-1057

9. MacDiarmid SA, Peters KM, Shobeiri SA et al (2010) Long-term durability of percutaneous tibial nerve stimulation for the treatment of overactive bladder. J Urol 183(1):234-240 
10. Vandoninck V, Van Balken MR, Finazzi AE et al (2003) Posterior tibial nerve stimulation in the treatment of idiopathic nonobstructive voiding dysfunction. Urology 61(3):567-572

11. Kim SW, Paick JS, Ku JH (2007) Percutaneous posterior tibial nerve stimulation in patients with chronic pelvic pain: a preliminary study. Urol Int 78:58-62

12. Van Balken MR, Vergunst $H$, Bemelmans BL (2006) Prognostic factors for successful percutaneous tibial nerve stimulation. Eur Urol 49(2):360-365

13. Capitanucci ML, Camanni D, Demelas F et al (2009) Long-term efficacy of percutaneous tibial nerve stimulation for different types of lower urinary tract dysfunction in children. J Urol 182(Suppl 4):2056-2061

14. De Gennaro M, Capitanucci ML, Mastracci P et al (2004) Percutaneous tibial nerve neuromodulation is well tolerated in children and effective for treating refractory vesical dysfunction. J Urol 171(5):1911-1913
15. Congregado Ruiz B, Pena Outeirino XM, Campoy Martinez P et al (2004) Peripheral afferent nerve stimulation for treatment of lower urinary tract irritative symptoms. Eur Urol 45:65-69

16. Doyle S, Carpenter A, Mcandrew F et al (2009) A comprehensive evaluation of children experiencing percutaneous tibial nerve stimulation (PTNS) for overactive bladder (OAB) refractory to behavior and pharmacological treatments. J Pediatr Urol 5:S100-S101

\section{Publisher's Note}

Springer Nature remains neutral with regard to jurisdictional claims in published maps and institutional affiliations.

\section{Submit your manuscript to a SpringerOpen ${ }^{\circ}$ journal and benefit from:}

- Convenient online submission

- Rigorous peer review

- Open access: articles freely available online

- High visibility within the field

- Retaining the copyright to your article

Submit your next manuscript at $\boldsymbol{\nabla}$ springeropen.com 